\title{
Ranking Objects Based on Relationships and Fixed Associations
}

\author{
Albert Angel \\ University of Toronto \\ albert@cs.toronto.edu
}

\author{
Surajit Chaudhuri \\ Microsoft Research \\ surajitc@microsoft.com \\ Nick Koudas \\ University of Toronto \\ koudas@cs.toronto.edu
}

\author{
Gautam Das \\ University of Texas at Arlington \\ gdas@cse.uta.edu
}

\begin{abstract}
Text corpora are often enhanced by additional metadata which relate real-world entities, with each document in which such entities are discussed. Such relationships are typically obtained through widely available Information Extraction tools. At the same time, interesting known associations typically hold among these entities. For instance, a corpus might contain discussions on hotels, cities and airlines; fixed associations among these entities may include: airline A operates a flight to city $\mathrm{C}$, hotel $\mathrm{H}$ is located in city $\mathrm{C}$.

A plethora of applications necessitate the identification of associated entities, each best matching a given set of keywords. Consider the sample query: Find a holiday package in a "pet-friendly" hotel, located in a "historical" yet "lively" city, with travel operated by an "economical" and "safe" airline. These keywords are unlikely to occur in the textual description of entities themselves, (e.g., the actual hotel name or the city name or the airline name). Consequently to answer such queries, one needs to exploit both relationships between entities and documents (e.g., keyword "pet-friendly" occurs in a document that contains an entity specifying a hotel name $\mathrm{H}$ ), and the known associations between entities (e.g., hotel $\mathrm{H}$ is located in city C).

In this work, we focus on the class of "entity package finder" queries outlined above. We demonstrate that existing techniques cannot be efficiently adapted to solve this problem, as the resulting algorithm relies on estimations with excessive runtime and/or storage overheads. We propose an efficient algorithm to process such queries, over large corpora. We devise early pruning and termination strategies, in the presence of joins and aggregations (executed on entities extracted from text), that do not depend on any estimates. Our analysis and experimental evaluation on real and synthetic data demonstrates the efficiency and scalability of our approach.
\end{abstract}

\section{INTRODUCTION}

In many application domains, such as e-commerce, social networking sites, digital libraries and collaborative knowledge repos-

Permission to copy without fee all or part of this material is granted provided that the copies are not made or distributed for direct commercial advantage, the ACM copyright notice and the title of the publication and its date appear, and notice is given that copying is by permission of the ACM. To copy otherwise, or to republish, to post on servers or to redistribute to lists, requires a fee and/or special permissions from the publisher, ACM. EDBT 2009, March 24-26, 2009, Saint Petersburg, Russia. Copyright 2009 ACM 978-1-60558-422-5/09/0003 ...\$5.00 itories, to name a few, metadata relate (unstructured) textual documents to the real-world entities discussed in them. For instance, in Wikipedia, the well-known collaborative encyclopædia, the unstructured document of an article about a person is related to entities such as the person, a birthplace, past employment institutions etc. In other domains, such as news articles, blog posts, etc. such document-entity relationships can be obtained through widely available Information Extraction tools ([4], [1]), which automatically identify named entities discussed in a document (e.g. Person, City, Company, Product).

Such entities are related via fixed associations typically known among them. For instance, a table in a relational database can associate neighbourhoods, houses for sale, and schools, via their location, thus giving rise to packages of associated entities (in this case, home-neighbourhood-school packages). Such information can be retrieved from several sources, e.g. from corporate databases, a collaborative public knowledge repository such as Freebase [8], etc. Moreover, it can be either static (e.g. school A is located in neighbourhood B) or dynamic (e.g. flights X and hotel $\mathrm{Y}$ are currently offered as a discounted holiday package).

In several instances, the goal is to identify packages whose entities each best match a given set of keywords. For instance, using comments from real-estate listings, blogs, etc., one may wish to identify a "Victorian", "3 bedroom" house for sale, located in a "low-crime", "safe" neighbourhood, that has a school with "bilingual education" and a renown "swimming team". As another example, using reviews from a travel planning site, one might wish to identify a holiday package, consisting of a destination that is generally considered to be "historical" and "lively", a hotel that is "pet-friendly", and travel operated by an airline renown for its "economical" and "safe" flights. Let us examine this motivating example of finding personalized holiday packages in greater detail.

Consider a corpus of reviews taken from a travel-planning site, shown in fig. 1. We assume that entities, such as hotels, cities and airlines, have been automatically identified in individual reviews, using a Named Entity Extraction tool. Moreover, we assume a relation containing known associations between entities, e.g. that hotel $\mathrm{H}$ is located in city $\mathrm{C}$, or that airline A flies to city $\mathrm{C}$. This relation can be available as a database table in the travel-planning site's infrastructure, or it can be retrieved from some external source (e.g. [8]). Furthermore, some parts of it might change very rarely, if at all (e.g. hotel $\mathrm{H}$ is located in city $\mathrm{C}$ ), whereas others can be subject to frequent updates (e.g. a discount holiday package containing a flight by airline $\mathrm{A}$, and a stay at hotel $\mathrm{H}$, is currently being offered). Our database thus consists of documents, representing reviews, and entities of three types, Hotels, Cities and Airlines. Relationships between documents and entities are represented by three tables of 


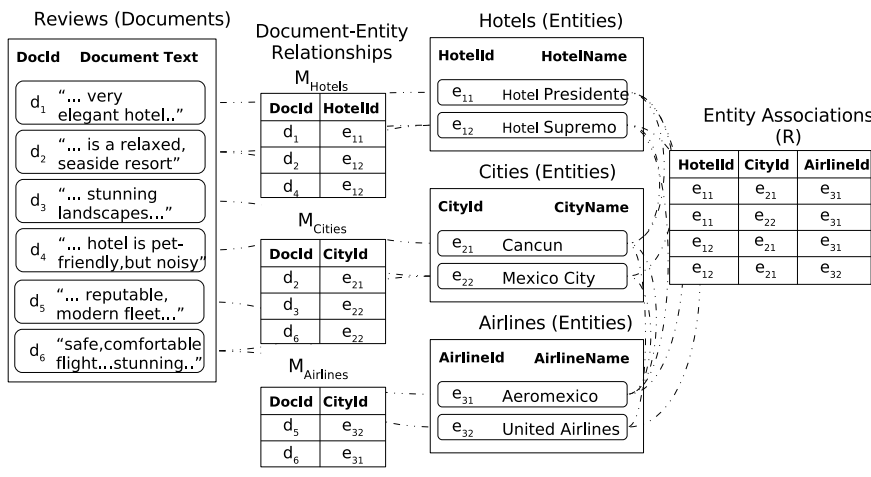

Figure 1: Data model

(DocId, EntId) tuples, one table for each entity type. (For instance, a tuple $(d, e)$ in table Hotels signifies that the review with id $d$ discusses the hotel with id $e$ - i.e. $e$ has been extracted as a topic of discourse from the text of $d$ ). Moreover, known associations between entities can be represented in a variety of ways, e.g. three tables denoting pairwise associations, etc. We abstract their representation by assuming a view $R$ over them. $R$ contains $\left(e_{1}, e_{2}, e_{3}\right)$ package tuples, signifying that hotel $e_{1}$, city $e_{2}$ and airline $e_{3}$ are associated, i.e. hotel $e_{1}$ is located in city $e_{2}$, airline $e_{3}$ flies to city $e_{2}$, and a flight with airline $e_{3}$ can be combined in a holiday package with a stay at hotel $e_{1}$. In fig. 1, a user might want to poll the public opinions, expressed in reviews, to find a holiday package in a city that is "historical", yet "lively", with a stay at a hotel that is "pet-friendly", and with travel operated by an "economical" and "safe" airline. These descriptive terms are unlikely to occur within the names of cities, hotels, or airlines; thus standard keyword search techniques cannot be used to answer such queries. Moreover, a user is not likely to be interested in all possible answers to their queries, but would prefer to be presented only with a small number of the most relevant results. We term this type of queries as Entity Package Finder queries (EPF). The necessary data to support such queries is a collection of:

1. Documents , which are searched by keywords;

2. Entities, each having a Type attribute (e.g. in fig. 1 Hotel Presidente is an entity of type Hotel);

3. Relationships between documents and entities, expressed as document-entity pairs, and denoting that a document refers to an entity; they can be static or dynamic; and

4. Associations among entities of different types, expressed as a relation containing tuples of entities; we refer to such tuples as packages

The aim in entity package finder queries is to identify the $\mathrm{k}$ most relevant packages of entities, with respect to each entity best matching a given set of keywords. This gives rise to the need to score packages, entities and documents with respect to the given keyword query. The score of a document wrt. a keyword query can be assessed using standard Information Retrieval techniques, such as textual similarity [17]. Note that entities are typically not directly related to keywords; for instance, named entity extraction from a document is typically based on more complex features than the simple presence of certain keywords [4]. For this reason, we will say that an entity matches a keyword query if it is related to documents that match the query. The quality of this match will depend both on the number of documents matching the keywords, that are related to the entity, as well as on the strength of the match between each such document and the given keywords. Thus, the score of the entity will be an aggregate of the scores of all documents matching the given keywords that are related to it. For example, in fig. 1, the score of $e_{12}$ (Hotel Supremo) wrt. keyword query "relaxed" will be an aggregate of the scores of documents $d_{2}, d_{4}$ wrt. the keyword "relaxed". Finally, to capture the notion that relevant packages are those that contain, overall, relevant entities, packages are scored based on an aggregate of their entities' scores (e.g. in fig. 1, the score of the holiday package $\left(e_{11}, e_{21}, e_{31}\right)$ wrt. some query will be an aggregate of the scores of $e_{11}, e_{21}$ and $e_{31}$ wrt. the query). In this work we use a scoring framework that encompasses a wide class of scoring semantics.

The essence of the entity package finder problem is to find the top-k packages with the highest scores, that result from score $a g$ gregations over documents matching a given query, and subsequent joins between entities of different types, according to a set of fixed associations. The techniques we propose are applicable for general entity finder queries, on any domain where documents can be searched by keywords (e.g. textual corpora, multimedia databases, medical databases etc.). In the following, solely for clarity of presentation, we will restrict ourselves to the case of text corpora.

Whereas this problem can be solved using standard RDBMS technology, the resulting solution would be highly inefficient. This is due to the fact that, in such a scenario, the precise scores of every entity and package would need to be calculated, followed by a selection of the top-k packages. Instead, early termination and pruning techniques can be used, to drastically reduce the necessary processing effort.

Adapting existing such techniques to this problem also leads to inefficient algorithms, as they require estimations with impractical runtime and/or storage overhead (see sec 3 ). To overcome this difficulty, we utilize the following intuition: Top scoring packages are expected to receive most of their score from top scoring entities, which, in turn, are expected to receive most of their score from top scoring documents. In our approach, documents are processed in descending score order, with respect to the given keyword query. Using document-entity relationships and entity associations, the aggregate scores of entities and packages are computed, incrementally, and in a rank-aware fashion. Periodically tight bounds on these scores are computed, and used to prune entities and packages that cannot rank among the final top-k. Thus, our algorithm is able frequently to terminate after processing only a small fraction of the input.

Our main contributions in this paper can be summarized as follows:

- We introduce the Entity Package Finder type of queries, able to answer useful questions with intuitive semantics.

- We formalize the EPF problem in a threshold algorithm framework, and demonstrate that existing techniques cannot be efficiently adapted to solve this problem, as the resulting algorithm relies on estimations with impractical overhead (runtime, storage).

- We propose a complementary early pruning/stopping approach, which interleaves rank-join and aggregation, and overcomes the need for such estimates. Our approach exploits all available knowledge regarding possible entity packages to provide tight bounding on package scores, leading to increased pruning efficiency.

- We analytically and experimentally evaluate the performance 
of our algorithm, on both real and synthetic data, and demonstrate its efficiency and scalability.

The rest of the paper is organized as follows: We formalize the EPF problem, and provide a threshold algorithm framework for it in section 2. We demonstrate the inapplicability of existing techniques (section 3), and propose an efficient algorithm in section 4. We discuss extensions to our algorithm and problem in section 4.2. The efficiency and scalability of our algorithm is demonstrated by analysis (section 5.1), as well as thorough experimental evaluation (section 5.2), on both real and synthetic data. We review other related work in section 6 , and conclude in section 7 .

\section{A FRAMEWORK FOR EPF}

Given the entity package finder class of queries introduced above, we subsequently formalize our problem, and describe a threshold algorithm framework for processing such queries.

\subsection{Data model}

Every document (e.g. review, blog post, etc.) in the corpus, is associated with a document id $d$. Every document contains search terms (keywords) (e.g. $d_{1}$ in fig. 1 contains keywords "very", "elegant" and "hotel", among others), and every document-term pair is associated with a score. These scores capture the importance of the term in the document, and can be derived using standard IR measures [17]. We assume the availability of an inverted index over the document corpus, i.e., for every search term, there exists an efficient way to retrieve all documents containing it, in descending score order.

Additionally, real-world entities are represented in our corpus. Every entity has an entity id $e$, and belongs to one of a number of types $T_{1}, T_{2}, \cdots, T_{n}$. For instance in fig. 1 Hotel Presidente is an entity of type Hotel. In addition, $n$ relational tables $M_{T_{i}}$ contain the relationships holding between documents and entities of type $T_{i}$. For example, in fig. 1, table $M_{\text {Hotels }}$ contains tuples of the form $(d, e)$, signifying that document $d$ mentions hotel $e$.

Finally, fixed associations between entities are available. Associations can be stored in a variety of ways (e.g. one or more relational tables), and can be either static, or dynamic (i.e. frequently updated). In either case, we assume the existence of a view, $R$ over this data. $R$ is an $n$-ary relation, with domain $T_{1} \times \cdots \times T_{n}$, and describes the union of all valid query answers: every tuple $\left(e_{1}, \cdots, e_{n}\right) \in R$, termed a package, contains associated entities. E.g., in fig.1, entities $e_{11}, e_{21}$ and $e_{31}$ form a valid holiday package. As $R$ might not be materialized in its entirety, we only require that it be accessible through a restricted API, able to efficiently return the packages containing a given entity $e$ in a given type $T$.

In the following, we assume that all $M_{T_{i}}$ tables, as well as the information necessary to provide access to $R$, fit into main memory, noting that our algorithms can be extended to handle cases where these assumptions do not hold ${ }^{1}$.

\footnotetext{
${ }^{1}$ This is a reasonable assumption, even for large-scale corpora. A typical document discusses a small number of entities, and for every such relationship we only need to maintain a document id and an entity id. Thus, the total memory overhead of $M_{T_{i}}$ tables will be a small multiple of the number of documents. This is consistent with our empirical observations on a large scale corpus of real data (see sec 5.2, REAL corpus), where the memory overhead of $M_{T_{i}}$ tables was, on average, under 10 bytes per document. Moreover, we expect the base information behind $R$ to fit in main memory, due to the semantics of the entity package finder problem. This,too, is consistent with our empirical observations; the $R E A L$ corpus, for instance, contained $110 \mathrm{~K}$ associations, requiring under $1 \mathrm{MB}$ of main memory.
}

\subsection{Query and Result Model}

An entity package finder query is an $n$-ary tuple containing sets of search terms, i.e. a tuple of the form: $\left(W_{1}, W_{2}, \cdots, W_{n}\right)$, where every $W_{i}$ is of the form $W_{i}=\left\{w_{1}, w_{2}, \cdots\right\}$. For example, in the holiday package case, a query could be ( $\{$ "pet-friendly" $\}$, \{"historical", "lively"\}, \{"safe"\}), meaning that the user would like to find a package with a "pet-friendly" hotel, a "historical" and "lively" city, and a "safe" airline.

An answer to such a query is a valid package, such that each of its entities, $i$, is related to at least one document $d$, containing some keyword in $W_{i}$. More formally, an answer is a tuple $\left(e_{1}, e_{2}, \cdots, e_{n}\right) \in R$ such that, for all $i \in\{1,2, \cdots, n\}$, there exists a document $d$ containing a term $w \in W_{i}$, such that $\left(d, e_{i}\right) \in$ $M_{T_{i}}$. For instance, in fig. $1,\left(e_{11}, e_{22}, e_{31}\right)$ is a valid answer to the query ( $\{$ "elegant" $\},\{$ "stunning" $\},\{$ "safe" $\}$ ). Of course, the more keywords from $W_{i} d$ contains, and the more such documents each entity $e_{i}$ is related to, the higher the relevance of the answer to the query. Additionally, since users are typically interested only in seeing a small number, $k$, of the most relevant answers to a query, the need to score answers arises.

\subsection{Scoring Answers}

Answer scoring proceeds in two levels: Firstly, entities of the same type need to be scored, based on the degree to which the documents that are related to them, match the given search terms. Thereafter, packages need to be scored, based on the scores of the entities they consist of ${ }^{2}$.

Entities are scored using two functions, $F_{a g g r}$ and $F_{c o m b} . F_{a g g r}$ is used to aggregate the scores of multiple documents that contain a specific search term and that are related to an entity. $F_{c o m b}$ is then used to combine the aggregated scores of an entity for all search terms. For example, in fig. 1 assume that documents $d_{3}, d_{6}$ contain the term "stunning", with associated scores $s_{1}, s_{2}$, respectively, and document $d_{6}$ contains the term "safe", with associated score $s_{3}$. Since both these documents are related to entity Mexico City, its score wrt. keywords "stunning" and"safe" will be $F_{\text {comb }}\left(F_{\text {aggr }}\left(s_{1}, s_{2}\right), F_{\text {aggr }}\left(s_{3}\right)\right)$.

In the following, we assume that $F_{\text {aggr }}$ is distributive over append (i.e. $F_{\text {aggr }}\left(s_{1}, s_{2}, \cdots, s_{n}\right)=F_{\text {aggr }}\left(F_{\text {aggr }}\left(s_{1}, s_{2}, \cdots, s_{i}\right)\right.$, $\left.\left.F_{\text {aggr }}\left(s_{i+1}, \cdots, s_{n}\right)\right)\right)$ and subset monotonic (i.e. $\left\{s_{1}, \cdots, s_{i}\right\} \subseteq$ $\left.\left\{s_{1}^{\prime}, \cdots, s_{j}^{\prime}\right\} \Longrightarrow F_{\text {aggr }}\left(s_{1}, \cdots, s_{i}\right) \leq F_{\text {aggr }}\left(s_{1}^{\prime}, \cdots, s_{j}^{\prime}\right)\right)$, and that $F_{\text {comb }}$ is monotonic (i.e. $\forall i s_{i} \leq s_{i}^{\prime} \Longrightarrow F_{\text {comb }}\left(s_{1}, s_{2}, \cdots, s_{n}\right)$ $\left.\leq F_{\text {comb }}\left(s_{1}^{\prime}, s_{2}^{\prime}, \cdots, s_{n}^{\prime}\right)\right)$. These properties are by no means restrictive, as they hold for most scoring functions used in practice (e.g. sum, weighted sum, max, etc.).

We note that scoring can also proceed with $F_{\text {aggr }}$ being applied on the results of $F_{c o m b}$; this can be efficiently reduced to query processing in the framework initially described, by utilizing the TA-NRA algorithm ([10]). Using these frameworks, we are able to capture most interesting and practical semantics for scoring entities; for instance, we can require that each query keyword occurs in at least one document matching an entity, or that all such documents be considered as a single pseudo-document for the purpose of scoring the entity.

For the second task, of scoring packages based on their entities' scores, following common practice, we employ a monotonic function $F_{p k g}$. For instance, if for a given query the score of en-

${ }^{2}$ Packages are only scored based on the scores of their comprising entities, because, in the EPF setting, packages have been defined as embodying certain knowledge. Although our setting can be extended to "fuzzy packages" with an attached confidence value, and corresponding changes to the scoring framework, we do not further discuss such extensions in this work. 


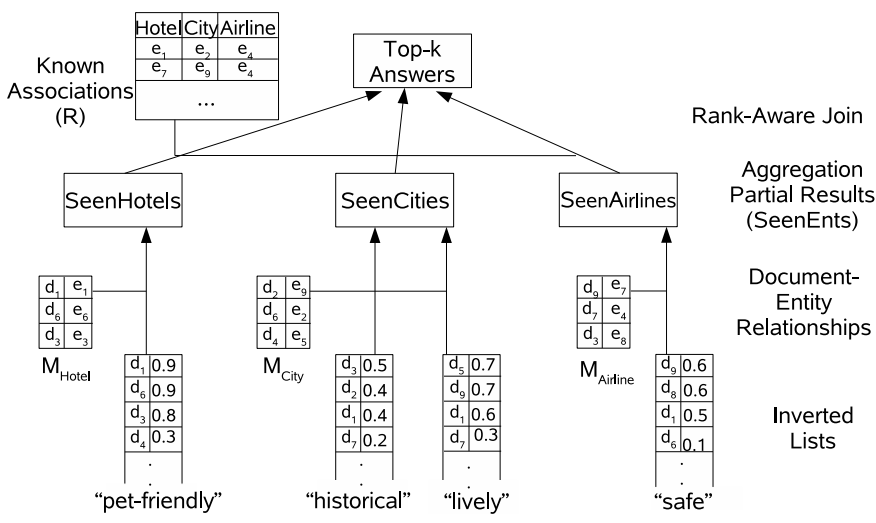

Figure 2: Execution Framework

tity Hotel Presidente is $s_{1}$, the one of Cancun is $s_{2}$, and that of Aeromexico is $s_{3}$, the score of the resulting holiday package will be $F_{p k g}\left(s_{1}, s_{2}, s_{3}\right)$.

\subsection{A Threshold Algorithm Framework}

We now present a general framework for processing entity package finder queries (fig.2).

As seen in fig. 2, processing is driven by sequential accesses on inverted lists, one for every keyword in the query. A list corresponding, e.g. to keyword "safe", consists of document ids for every document containing the word "safe", along with scores that denote their relevance to "safe", in descending score order. Every document id encountered is used to probe in-memory documententity relationship tables (cf. $M_{\text {Airline }}$ in fig. 2), so as to retrieve the entities related to the document. The document score and related entities are used to update the current known bounds on entity scores. Such information is maintained in the Aggregation Partial Result Tables, termed SeenEnts for brevity (cf. SeenAirlines in fig. 2); we describe their functionality shortly.

Using the available score bounds on entities, a partial ordering on them can be determined (e.g., if entity $e_{1}$ has a score in $[1,2]$, and the score of $e_{2}$ is in the interval [2.3,3.5], then clearly $e_{2}$ will have a higher score than $e_{1}$ ). Whenever a query processing algorithm decides that sufficient information wrt. entity scores is available (e.g. the top-3 Airlines, top-2 Cities and top-5 Hotels, and their scores are known), it can use this information to try and calculate the $k$ top-scoring packages. This is done by essentially performing a multi-way join among the top entities of each type, using probes to the in-memory known associations view, $R$, to evaluate the join condition. To avoid superfluous processing effort, a query processing algorithm should use a rank-aware join operator ([11]), allowing for earlier termination, without the need to consider all top-scoring entities. Such approaches are described in sec. 3 and sec. 4.

Computing Entity Aggregate Scores: For every entity type $T$, we maintain a table SeenEnt ${ }_{T}$ (cf. SeenCities in fig. 2), which captures the current level of knowledge wrt. the most promising entities in $T$. Specifically, for every entity $e$, SeenEnt $T_{T}$ maintains lower and upper bounds on its final score $(L B$ and $U B$, respectively). These bounds are used as in the Threshold Algorithm ([10]), for pruning entities guaranteed not to be part of the final answer, and for early termination. For reasons of efficiency, SeenEnt $t_{T}$ is indexed by entity id.

Computing Lower Bounds: In order to compute lower bound information, SeenEnt $t_{T}$ additionally records, for every entity $e$, the number of times a document related to $e$ has been encountered on every inverted list list $_{i}$, NumSeen $_{i}$, and the score that $e$ has received up to now from all documents on list $_{i}$, AgScore $_{i}$. Put differently, AgScore $_{i}$ is the score that $e$ would obtain from the keyword corresponding to list $_{i}$, if no other documents matching $e$ are found on list ${ }_{i}$. AgScore ${ }_{i}$ is a lower bound on the final score of $e$ wrt. the keyword corresponding to list $_{i}$, and the full final score of $e$ is lower bound by $L B=F_{\text {comb }}\left(\right.$ AgScore $_{1}$, AgScore $\left._{2}, \cdots\right)$.

Computing Upper Bounds: In order to calculate an upper bound, $U B$, on the score of entity $e$, we utilize, for every inverted list $l_{i s t}$, the following items of information. Firstly, the score of the last document retrieved from list $_{i}$, maxUnseen m $_{i}$; this is an upper bound on the score that any document, not yet retrieved from list $t_{i}$, can have. Secondly, we require knowledge of the maximum number of documents in list $t_{i}$ that can influence the score of $e$, which we term the cardinality of $e$ in list $_{i}, \operatorname{card}\left(e\right.$, list $\left._{i}\right)$; we shortly discuss ways of calculating it. The maximum further score $e$ can obtain from list $_{i}$ is maxFurther $_{i}=F_{\text {aggr }}\left(\right.$ maxUnseen $_{i}, \cdots$, maxUnseen $\left._{i}\right)$, where $F_{\text {aggr }}$ is applied on $\operatorname{card}\left(e\right.$, list $\left._{i}\right)$ variables with value maxUnseen $_{i}$. The maximum score $e$ can obtain from list $_{i}$ is maxTotal $_{i}=F_{\text {aggr }}($ AgScore $_{1}$, maxFurther $\left._{1}\right)$. Finally, an upper bound on the score of $e$ is $U B=F_{\text {comb }}\left(\operatorname{maxTotal}_{1}, \operatorname{maxTotal}_{2}, \cdots\right)$.

Computing Entity Cardinalities: Let us now discuss how the cardinality of an entity $e$ in a list list $_{i}$ can be calculated, by judiciously materializing information about certain entities. In a preprocessing phase, we scan all inverted lists, and calculate and store the ids and scores wrt. list ${ }_{i}$ of the $X$ entities with the highest cardinalities in $l_{i s t_{i}}$, as well as an upper bound, $\operatorname{maxCard}_{i}$, on the cardinality of all other entities in list $_{i}{ }^{3}$. By using this information at query time, for every entity $e$ and list list $_{i}$ we have knowledge of either i) a useful upper bound on the cardinality of $e$ in list $_{i}$ (namely, $\operatorname{maxCard}_{i}$ ), or ii) the precise score of $e$ wrt. list $_{i}$ (in this case, we record the score in the relevant SeenEnt table, and set the relevant NumSeen $_{i}$ to the special value "ALL"). This enables the calculation of the maximum score, MaxUnseen $_{T}$, obtainable by any entity $e$ of type $T$ that has not yet been seen (exploiting the the monotonicity properties of $F_{\text {aggr }}, F_{\text {comb }}$ ). MaxUnseen ${ }_{T}$ can be used to compute an early termination criterion. We note that this preprocessing has a low overhead ${ }^{4}$.

\subsubsection{Access Primitives}

Given the structures above, we define two forms of access that a query processing algorithm can use to access data: Sequential Accesses (SA's), and Batch Accesses (BA's) (the latter correspond to standard TA Random Accesses, optimized for the entity package finder setting).

A SA on an inverted list (alg. 1) essentially retrieves a block ${ }^{5}$ of document ids from the list (line 1), looks up entities related to these documents (line 2), and updates bounds on the scores of these entities (lines 3-8). At some point of time, an algorithm determines that a superset of all necessary entities of a type $T$ has been identified (i.e., any entity that does not have a corresponding entry in SeenEnt $t_{T}$ is not needed to identify the query answer). Thus, the

\footnotetext{
${ }^{3} X$ is a small number, so that storing these entities and their scores requires a negligible amount of storage; for instance, in our experiments we materialized $1 \%$ of all entities, resulting in an additional storage overhead of less than $0.01 \%$ the size of inverted lists.

${ }^{4}$ For instance, on a large, real dataset we used in our experiments (see sec. 5.2, corpus $R E A L$ ) this preprocessing requires on average less than a second per inverted list per entity type; similar trends were observed on larger synthetic datasets.

${ }^{5}$ For reasons of efficiency, and following common practice, documents are retrieved in blocks, as opposed to one-at-a-time.
} 

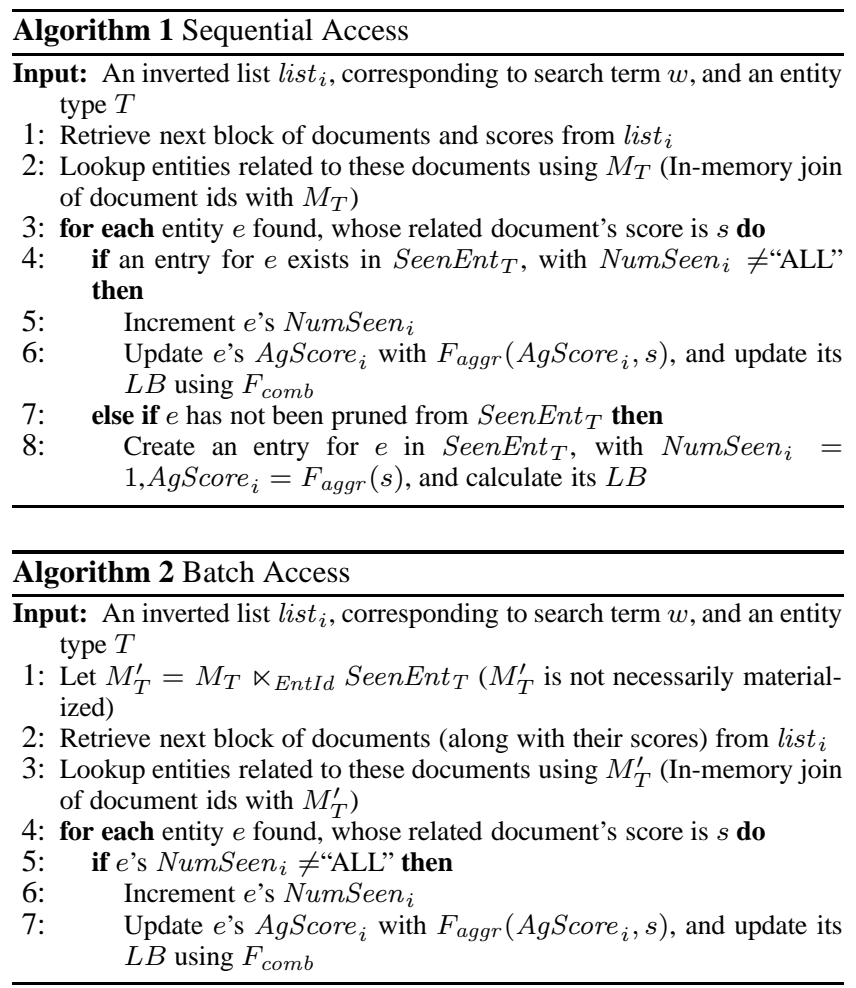

only necessary actions are to discover the actual scores of the required top entities, and to prune away the remaining ones.

For this purpose, Random Accesses on the scores of all candidate entities are needed. However, if one were to perform individual Random Accesses on the score of an entity, $e$, one would need to i) retrieve all documents related to $e$, and ii) scan them to determine their score wrt. all query keywords. These operations would entail a large number of disk random seeks. For instance, in a corpus of real data we used in our experiments (sec 5.2, corpus $R E A L$ ), on average 17-40 documents would have been read from disk per Random Access, depending on the entity's type. Hence, true Random Accesses are not a feasible option in our setting. Due to these performance considerations, we instead process Random Accesses in batches, using an access primitive we term Batch Access (BA) (shown in alg. 2).

A BA on an inverted list follows the steps we outlined above for SA's; however, it ignores documents that do not correspond to entities encountered so far. Thus, the result of a series of BA's on a list is precisely the same as that of performing random accesses on the scores of all entities wrt. the list, at a fraction of the cost.

\section{THE LAYERED ALGORITHM}

Given the above framework, we present the Layered algorithm (shown in alg. 3), a baseline approach for solving the entity package finder problem.

We define $D_{i}$ as the number of top-scoring entities of a type $T_{i}$ that are needed to identify the top-scoring packages. Assume that $D_{i}$ are known, for all $i$ (We will subsequently argue that this is an unrealistic assumption, and show how to alleviate it.). For instance, in fig. 2, assume it is known that, given the top $D_{1}$ Hotels, the top $D_{2}$ Cities and the top $D_{3}$ Airlines, along with their scores, it is possible to calculate the top $k$ holiday packages. Then, the top $k$ packages can be calculated by first identifying the top $D_{i}$ entities of each type $T_{i}$, and then using these to compute the top $k$ packages.

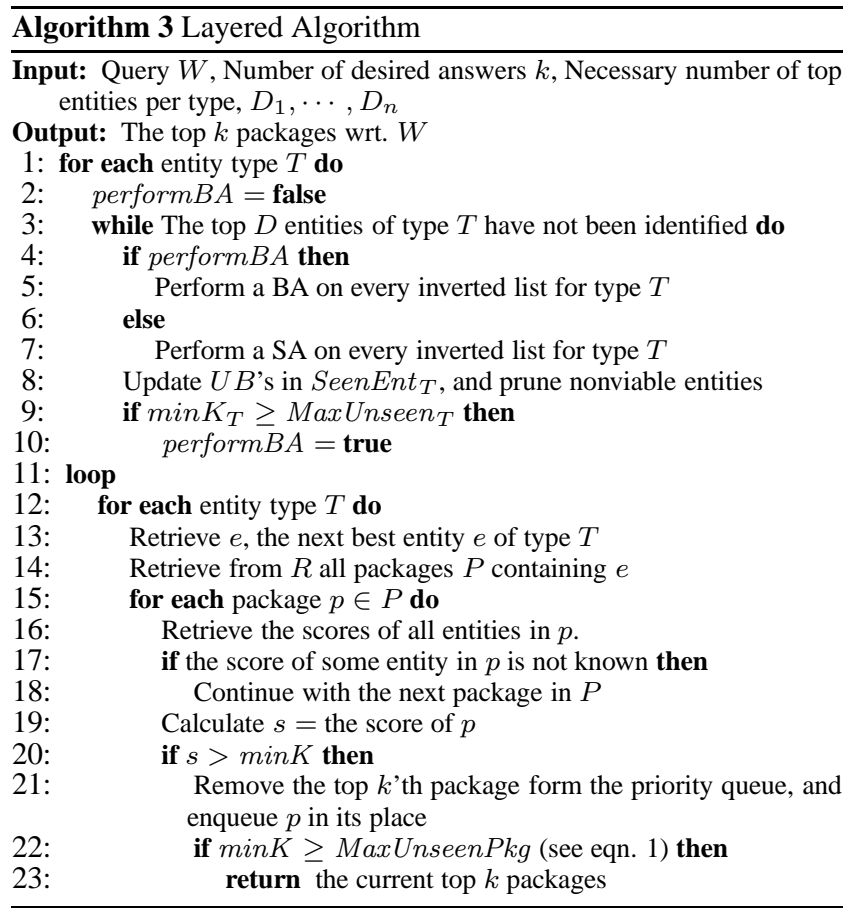

More specifically, the Layered algorithm (a high-level outline of which is shown in alg. 3), maintains a priority queue for every entity type $T_{i}$, containing the $D_{i}$ entities with the currently largest $L B$ 's. Let $\min K_{T_{i}}$ be the smallest $L B$ among these. Every entity of type $T_{i}$ with $U B<\min K_{T_{i}}$ can safely be pruned from SeenEnt $T_{T_{i}}$, as it is certainly not among the top $D_{i}$ entities of its type (line 8). Using this pruning criterion, Layered performs SA's on every inverted list until a superset of the top $D_{i}$ entities of each type $T_{i}$ have been identified (line 9); thereafter it performs BA's instead (line 10), until the top $D_{i}$ entities are identified.

Subsequently, a rank-join ([11]) algorithm is used to calculate the top packages (lines 11- 23). Layered iterates over every entity type $T$, selecting the next best entity $e$ of type $T$ (line 13). It then uses $e$ to probe the known associations table, $R$, and retrieve the packages containing $e($ line 14). For every such packages $p$, if the scores of all entities in $p$ are known, Layered calculates the exact score of $p$ (using $F_{p k g}$ ) (lines 16-19). Layered also maintains the current top $k$ packages in a priority queue, and the score of the current top- $k$ 'th package, $\operatorname{minK}$.

At any point of time, in order to decide whether the priority queue contains the true top $k$ packages, and thus it is safe to terminate processing, Layered utilizes the following observation, due to the monotonicity of $F_{p k g}$. Let $s_{i}(b)$ be the score of the top $b$ 'th entity of type $T_{i}$. After having examined the top $b_{i}$ entities of type $T_{i}$, the best score obtainable by a package that has not yet been encountered is

$$
\begin{aligned}
& \text { MaxUnseenPkg }=\max \left\{F_{p k g}\left(s_{1}\left(b_{1}\right), s_{2}(1), s_{3}(1), \cdots, s_{n}(1)\right)\right. \\
& \quad F_{p k g}\left(s_{1}(1), s_{2}\left(b_{2}\right), s_{3}(1), \cdots, s_{n}(1)\right) \\
& \left.\quad F_{p k g}\left(s_{1}(1), \cdots, s_{n-1}(1), s_{n}\left(b_{n}\right)\right)\right\}
\end{aligned}
$$

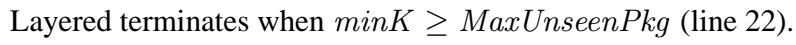

\subsection{Execution example}

To better illustrate the workings of Layered, we present the following execution example. We will utilize the sample corpus shown in table 3.1, and the following scoring functions: $F_{a g g r}\left(x_{1}, \cdots, x_{j}\right)=$ 
(a) Inverted List for keyword $w_{1}$

\begin{tabular}{|cc|}
\hline DocId & Score \\
\hline$d_{5}$ & 1.0 \\
$d_{3}$ & 0.8 \\
$d_{6}$ & 0.5 \\
$d_{8}$ & 0.2 \\
$d_{7}$ & 0.2 \\
\hline
\end{tabular}

(b) Inverted List for keyword $w_{2}$

(c) Inverted List

\begin{tabular}{|cc|}
\hline DocId & Score \\
\hline$d_{1}$ & 1.0 \\
$d_{7}$ & 0.9 \\
$d_{4}$ & 0.6 \\
$d_{2}$ & 0.2 \\
$d_{9}$ & 0.1 \\
\hline
\end{tabular}

for keyword $w_{3}$

\begin{tabular}{|cc|}
\hline DocId & Score \\
\hline$d_{13}$ & 0.7 \\
$d_{18}$ & 0.5 \\
$d_{15}$ & 0.2 \\
$d_{16}$ & 0.1 \\
$d_{10}$ & 0.1 \\
\hline
\end{tabular}

(e) Doc - Entity

(d) Doc - Entity relationships, relationships, $M_{T_{1}}$

\begin{tabular}{|c|c|c|c|c|c|c|c|}
\hline \multirow{2}{*}{\multicolumn{2}{|c|}{ DocId EntId }} & \multirow{2}{*}{\multicolumn{2}{|c|}{$\begin{array}{l}\text { DocId EntId } \\
\text { (cont'd) }\end{array}$}} & \multicolumn{2}{|c|}{ DocId EntId } & \multicolumn{2}{|c|}{ Entity type } \\
\hline & & & & $d_{10}$ & $\alpha$ & $T_{1}$ & $T_{2}$ \\
\hline$d_{1}$ & $\bar{b}$ & $d_{6}$ & $e$ & $d_{10}$ & $\gamma$ & $b$ & $\alpha$ \\
\hline$d_{2}$ & $b$ & $d_{7}$ & $a$ & $d_{13}$ & $\alpha$ & $a$ & $\gamma$ \\
\hline$d_{3}$ & $a$ & $d_{7}$ & $c$ & $d_{15}$ & $\gamma$ & $b$ & $\beta$ \\
\hline$d_{4}$ & $a$ & $d_{8}$ & $b$ & $d_{16}$ & $\alpha$ & $e$ & $\beta$ \\
\hline$d_{5}$ & $a$ & $d_{8}$ & $c$ & $d_{18}$ & $\beta$ & & \\
\hline$d_{5}$ & $b$ & $d_{9}$ & $c$ & & & & \\
\hline$d_{6}$ & $c$ & $d_{9}$ & $e$ & & & & \\
\hline
\end{tabular}

(g) Layered execution example, SeenEnt ${ }_{T_{1}}$

\begin{tabular}{|c|c|c|c|c|c|c|c|}
\hline Step & $\overline{\text { EntId }}$ & $\begin{array}{l}\text { NumSeen } \\
w_{1}\end{array}$ & $\begin{array}{l}\text { AgScore } \\
w_{1}\end{array}$ & $\begin{array}{l}\text { NumSeen } \\
w_{2}\end{array}$ & $\begin{array}{l}\mathrm{AgS} \\
w_{2}\end{array}$ & LB & UB \\
\hline \multirow{2}{*}{1} & $a$ & 1 & 1 & 0 & 0 & $\overline{0}$ & 3 \\
\hline & $b$ & 1 & 1 & 1 & 1 & 1 & 3 \\
\hline \multirow{3}{*}{2} & $a$ & 2 & 1.8 & 1 & 0.9 & 0.9 & 2.6 \\
\hline & $b$ & 1 & 1 & 1 & 1 & 1 & 2.6 \\
\hline & $c$ & 0 & 0 & 1 & 0.9 & 0 & 2.4 \\
\hline \multirow{4}{*}{3} & $a$ & 2 & 1.8 & 2 & 1.5 & 1.5 & 2.1 \\
\hline & $b$ & 1 & 1 & 1 & 1 & 1 & 2 \\
\hline & $c$ & 1 & 0.5 & 1 & 0.9 & 0.5 & 1.5 \\
\hline & $e$ & 1 & 0.5 & 0 & 0 & 0 & 1.5 \\
\hline \multirow{4}{*}{4} & $a$ & 2 & 1.8 & 2 & 1.5 & 1.5 & 1.7 \\
\hline & $b$ & 2 & 1.2 & 2 & 1.2 & 1.2 & 1.4 \\
\hline & $c$ & 2 & 0.7 & 1 & 0.9 & 0.7 & 0.9 \\
\hline & $e$ & 1 & 0.5 & 0 & 0 & 0 & 0.6 \\
\hline
\end{tabular}

Table 1: Sample corpus and Layered execution example

$\sum x_{l}, F_{\text {comb }}\left(x_{1}, \cdots, x_{j}\right)=\min \left(x_{l}\right), F_{p k g}\left(x_{1}, \cdots, x_{n}\right)=\sum x_{l}$ if $\min \left(x_{l}\right)>0$, and 0 otherwise. For the sake of clarity, we assume that, during preprocessing, no entity scores were materialized, and the bound computed on the maximum cardinality of all entities wrt. any list is 3 (i.e., in this corpus, the score of every entity can be influenced by at most 3 documents). Moreover, we assume that SA's access inverted lists one document id at a time, even though in practice, for performance considerations, document id's are retrieved in larger batches.

Assume we want to identify the top 1 package wrt. query $\left(\left\{w_{1}\right.\right.$, $\left.\left.w_{2}\right\},\left\{w_{3}\right\}\right)$; i.e., the top package where the first entity (of type $T_{1}$ ) is most relevant to keywords $w_{1}$ and $w_{2}$, and the second entity (of type $T_{2}$ ) is most relevant to $w_{3}$. Moreover, assume Layered has somehow determined that $D_{1}=3$ and $D_{2}=2$ top entities of each type, $T_{1}$ and $T_{2}$, are needed in order to determine the top 1 package.

Layered will first identify the top $D_{1}=3$ entities of type $T_{1}$ (lines 3-10). A SA is performed on both inverted lists of $w_{1}$ and $w_{2}$ (line 7). This results in documents $d_{5}$ and $d_{1}$ being retrieved. By probing $M_{T_{1}}$, Layered discovers that they are related to entities $a, b$ and $b$, respectively. Entries for $a$ and $b$ are created in
SeenEnt $T_{T_{1}}$; information regarding how many times they have been encountered on every list, and their aggregate score wrt. every list, is recorded. $a$ initially has lower bound $L B=F_{\text {comb }}(1,0)=0$, since, at this point, it is possible that its score wrt. $w_{2}$ will be 0 (recall $F_{\text {comb }}=\min$ ). Upper bounds for all entries in SeenEnt $t_{T_{1}}$ are then calculated, (line 8) using their current AgScore's, and ev-

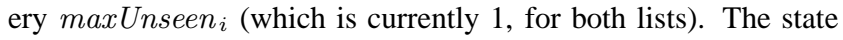
of SeenEnt $T_{T_{1}}$ after these operations is shown in table $0(\mathrm{~g})$, step 1. This procedure is repeated another three times (tab. $0(\mathrm{~g})$, steps 24). Moreover, after step 4, the best score that entity $e$ can possibly obtain $(U B=0.6)$ is less than the worst possible score that any of the top- 3 entities $a, b$ or $c$ can obtain; as only 3 top entities need to be calculated, $e$ is pruned (line 8). Independently, since no further entity yet unseen can become part of the top-3 (line 9), Layered proceeds by performing BA's on all lists, until the final scores of $a, b$ and $c(1.5,1.2$ and 0.9 , respectively) are discovered. Similarly, Layered identifies that the top $D_{2}=2$ entities of type $T_{2}$ are $\alpha$ and $\beta$, with scores 0.9 and 0.5 , respectively.

Layered then proceeds by joining the identified top entities of types $T_{1}$ and $T_{2}$, using known associations table $R$, in a rank-aware fashion (lines 11-23). Using $R$, after processing entities $a, b$ and $\alpha$, Layered determines that $b, \alpha$ is the currently top package, with score 2.1. Moreover, the next best entities of each type have scores 0.9 and 0.5 , respectively. Thus, assuming they were associated with the top entities of the other type, the resulting packages would have scores $F_{p k g}(0.9,0,9)=1.8$ and $F_{p k g}(1.5,0,5)=2$, respectively. Since none of these is better than the score of the package $b, \alpha$, Layered concludes that it is the top 1 package, and terminates processing (line 22).

\subsection{Estimating $D_{i}$}

The Layered algorithm crucially depends on knowing the precise value of all $D_{i}$ (the number of entities of every type $T_{i}$ that are needed, in order to identify the top $k$ packages). Clearly, this is an unrealistic assumption, as in general one would need to execute the entity package finder query in its entirety in order to determine these values. Thus, a realization of the Layered algorithm would call for an estimation $D_{i}^{\prime}$ of $D_{i}$. Let us now examine the implications on query processing, by showing how the $D_{i}^{\prime}$ estimation can be performed using state-of-the art techniques.

This estimation problem is strongly related to the Depth Estimation problem in the context of a rank-join algorithm ([16]). Indeed, [16] proposes techniques that could be readily adapted for estimating $D_{i}^{\prime}$. However, this adaptation necessitates a strong assumption; namely that sufficient statistics are known about the distribution of the scores of packages wrt. any given query. The statistics should be detailed enough to provide the following information, as accurately as possible:

Given a tuple of entity scores $\left(s_{1}, s_{2}, \cdots, s_{n}\right)$,

- How many valid packages $\left(e_{1}, e_{2}, \cdots, e_{n}\right)$ exist, such that the score of each $e_{i}$ is precisely $s_{i}$ ? and

- For every $i$, what is the largest number $s_{i}^{\prime}$ such that $s_{i}^{\prime}<$ $s_{i}$, and there exists at least one valid package with score $\left(s_{1}, \cdots, s_{i-1}, s_{i}^{\prime}, s_{i+1}, \cdots, s_{n}\right)$ ?

In order to provide such statistics, one has essentially two options. Firstly, one can materialize in a preprocessing phase, for every entity of every type, the precise distribution of the scores of its related documents wrt. every inverted list. These distributions can then be used, in conjunction with the known associations table, to estimate the score distribution of packages at runtime. However, materializing all this information has an enormous space overhead, 
on the order of the corpus size itself ${ }^{6}$.

The second option, is to estimate the score distribution of packages by sampling (assuming the API available on $R$ enables such an operation, e.g. in the case of dynamic known associations). Specifically, at runtime, one can select some packages at random, and calculate exactly the scores of all their entities, wrt. the given query. Unfortunately, these calculations have a very high runtime overhead, as they require Random Accesses on the scores of a number of entities, which are unfeasibly expensive operations in our setting $^{7}$ ). Moreover, in order to ensure that the sampling produces a reasonably small estimation error, a sizeable fraction of entities' scores would need to be calculated. Hence, the runtime overhead of this option is clearly too high to be used in practice.

Summarily, using state-of-the art techniques for estimating $D_{i}^{\prime}$ in the context of the Layered algorithm, will either have an excessive storage and/or runtime overhead, or will entail sizeable and unpredictable estimation errors. These errors will largely affect the performance of the Layered algorithm. If, for some $i, D_{i}^{\prime}>D_{i}$, Layered will be forced to perform more processing than necessary. Even worse, if for some $i, D_{i}^{\prime}<D_{i}$, Layered will not be able to identify the top $k$ packages; thus it will need to perform some additional processing (including re-doing some processing previously performed), to first identify a number $D_{i}^{\prime \prime}>D_{i}^{\prime}$ of top entities of type $T_{i}$, and then resume the rank-join process.

It should thus be clear that an efficient realization of Layered is not possible, due to the significant overheads imposed by the estimation procedure. To address this issue, we subsequently introduce Interleaved, an efficient algorithm for the entity package finder problem, that does not rely on knowledge or estimations of $D_{i}$. Despite not using such estimates, we show that Interleaved is as efficient as a hypothetical, idealized instantiation of the Layered algorithm, that would have freely available accurate knowledge of all $D_{i}$.

\section{THE INTERLEAVED ALGORITHM}

The essense of the Interleaved algorithm is to incrementally maintain score information on the package level. To do so, while scanning inverted lists, Interleaved keeps updating the score bounds of both entities, as well as packages (by interleving the operations of rank-aware aggregation and rank-join). Using this knowledge, it is able to prune non-promising entities and packages, and thus to terminate early. The high efficiency of Interleaved originates principally from two novel features: the interleaved pruning of entities, and the tight bounding of package scores. Both these features are designed to exploit the distribution of fixed associations, and

\footnotetext{
${ }^{6}$ In a large-scale, real dataset utilized in our experiments (see sec. 5.2 , corpus $R E A L$ ), storing such information incurs an additional average space overhead of $15 \%$ per entity type. The corpus contains 9 entity types, so these statistics require more storage than the corpus itself. A similar space blowup was observed in the synthetic corpora we utilized in our experiments.

${ }^{7}$ With respect to the cost of Random Accesses, note that, in order to calculate the score of a single entity, one would need to retrieve all documents in the corpus that are related to the entity, and scan them to determine their score wrt. all query keywords. Thus, this calculation entails a number of disk random seeks equal to the cardinality of the entity (i.e. the number of documents related to it); in a corpus of real data we used in our experiments ( $\mathrm{sec} 5.2$, corpus $R E A L$ ), this was on average 17-40 documents per entity (depending on the type of the entity). Note also that such Random Accesses cannot be performed in batches - i.e. using Batch Accesses- as this would result in redundantly executing a large part of the query itself in a preprocessing phase, solely for the purpose of obtaining estimates.
}

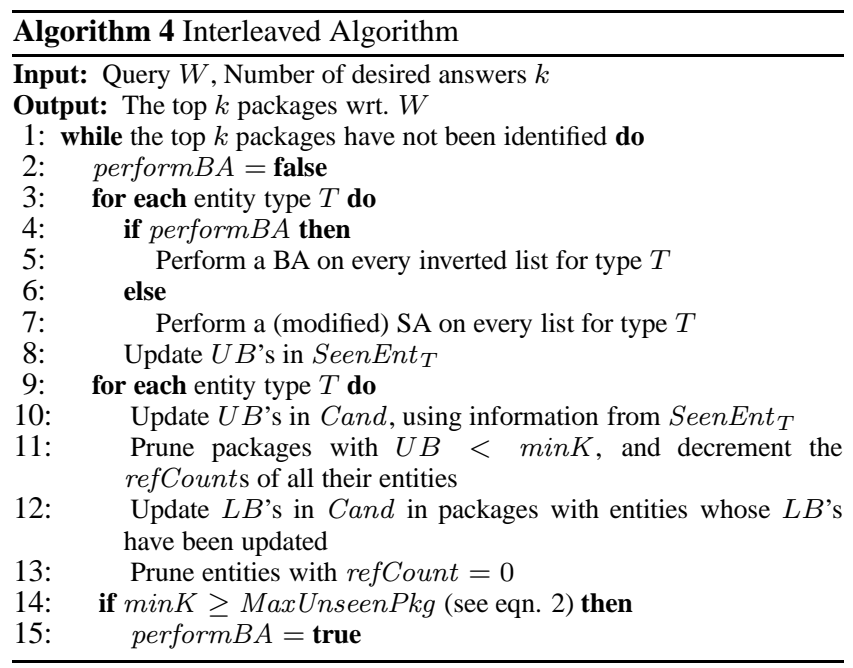

are subsequently explained in detail. Through careful engineering, this added functionality is achieved with only moderate extra bookkeeping effort.

Interleaved maintains a few additional data structures, besides those detailed in section 2.4. Firstly, a Candidate Package table, Cand, which contains all promising packages (i.e. those that might rank among the final top-k). Entries in Cand are of the form $\left(e_{1}, e_{2}, \cdots, e_{n}, U B, L B\right)$, where $e_{i}$ denote pointers to the entry of entity $e_{i}$ in the respective SeenEnt $T_{T_{i}}$ table; $U B$ and $L B$ are bounds (upper and lower, respectively) on the final score of the package. Interleaved also maintains a priority queue containing the $k$ most promising packages (based on their $L B$ 's), as well as $\min K$, the $L B$ of the $k$ 'th top package. Finally, entries in SeenEnt tables are augmented with a reference count field, refCount (this field is used for interleaved pruning, and is described shortly).

Let us now examine the operation of Interleaved (pictured in alg. 4 ) in detail. Until the top- $k$ packages have been identified, Interleaved does the following (line 1). First, it performs some SA's on every inverted list, for every entity type (line 7). The algorithm for SA's it uses is slightly modified from the alg. 1. Specifically, whenever an entity $e$ is first encountered (line 7 in alg. 1), entries in $C a n d$ are created for all the packages $e$ participates in. This is done by probing the known associations table, $R$, for packages containing $e$, and creating $C a n d$ entries for every such package. If needed, placeholder entries are created in SeenEnt tables for other entities in these packages; subsequently, the refCounts of all entities in these packages are incremented.

Having performed these modified SA's, Interleaved updates upper bound information in SeenEnt tables (line 8). Using this information, it then updates upper bounds in the Candidate Package table (line 10). Interleaved prunes packages that are certain to not rank among the final top-k, based on their score upper bound, and decrements their entities' refCounts (line 11). Interleaved also updates lower bound information in Cand (line 12); for the sake of efficiency, it only updates packages containing entities with recently updated $L B$ 's (this is recorded in a separate flag).

In line 13, the feature of interleaved pruning manifests itself. Entities that do not participate in any promising package are pruned. In this manner, a large number of entities can be pruned, independently of how high their score is, just by exploiting the distribution of known associations. This leads to a significant decrease in processing effort, as there are fewer entities to keep track of, and to earlier termination. Moreover, by keeping track of the number 
of packages an entity participates in (through its refCount), interleaved pruning is very efficient, and imposes but a minimal storage overhead.

Finally, in line 14, Interleaved decides whether a superset of the final top $k$ answers has been identified, and SA's can therefore be replaced by BA's. Another significant feature of Interleaved is an improved condition for making this decision. Adapting standard rank-join techniques ([11]), as in the case of Layered, results in an overly conservative bound on the maximum score of a package not yet encountered. Specifically, let $s_{i}\left(b_{i}\right)$ be the best score obtainable by an entity of type $T_{i}$ that has not yet been encountered, and let $s_{i}(1)$ be the overall best score obtainable by an entity of type $T_{i}$. Then, the bound on the best score obtainable by a package that has not yet been encountered is given by eqn. 1 . This bound corresponds to assuming that a package may exist, containing the top entity of each type but one, and an entity yet unseen on that type. Interleaved, on the other hand, by maintaining bounds on the scores of all packages that contain at least one seen entity, has access to more information; namely that any package not in Cand can only consist of entities not yet encountered. Hence, Interleaved uses the following upper bound on the score of a package not in Cand in line 14:

$$
\text { MaxUnseenPkg }=F_{p k g}\left(\text { MaxUnseen }_{T_{1}}, \cdots, \text { MaxUnseen }_{T_{n}}\right)
$$

This bound, which exploits known associations, is in fact a tight bounding scheme on package scores (in the spirit of [15]); in a sense, this is the tightest possible bound that can be provided. More formally, there always exists a set of documents that could appear later on some inverted lists, and a set of their relationships to entities in the corpus, such that the most promising package not currently in Cand obtains a final score equal to MaxUnseenPkg. This is in contrast to the bound employed by Layered and by rank-join algorithms, which can be significantly looser. The tight bounding scheme significantly boosts the pruning power of Interleaved.

To summarize, in Interleaved we have effectuated an early pruning and termination strategy, that is entirely independent of any estimates, and has a very moderate bookkeeping overhead. Entities are now pruned based on whether they might be part of some top package, rather than their current score alone, by dint of an interleaved pruning policy that exploits the distribution of packages. Moreover, in contrast with standard rank-join algorithms, package scores are tightly bounded, based on the best score they can actually achieve. These features offer the Interleaved algorithm additional opportunities for improved performance, compared to Layered, without relying on potentially erroneous and expensive estimations.

\subsection{Execution example}

To illustrate the workings of Interleaved, we show how it processes the scenario previously discussed in sec. 3.1; we refer to table 3.1 for the corpus used. In table 4.1 we provide a trace of the contents of the Cand table at every execution step (i.e., at every iteration of line 1). Moreover, as the functionality of SeenEnt tables has been demonstrated in sec 3.1 , we only provide a summary thereof, containing $L B$ 's and $U B$ 's for every entry. In step 1, a SA is performed on every inverted list (line 7), and SeenEnt tables are populated. Moreover, entries concerning all packages that contain an entity in some SeenEnt table are inserted in Cand. Finally, empty entries are inserted in SeenEnt tables, concerning entities that are mentioned in some package in Cand, but do not have a SeenEnt entry (e.g. $\beta, \gamma$ ). This procedure is repeated in step 2.

\begin{tabular}{|l|lll|lll|lllll|}
\multicolumn{4}{c}{} & \multicolumn{1}{c}{ SeenEnt $_{T_{1}}$} & SeenEnt $_{T_{2}}$ & \multicolumn{4}{c|}{ Cand } \\
\hline Step & EntId $L B$ & $U B$ & EntId $L B$ & $U B$ & EntId $_{1}$ & EntId $_{2}$ & $L B$ & $U B$ \\
\hline 1 & $a$ & 0 & 3 & $\alpha$ & 0.7 & 2.1 & $b$ & $\alpha$ & 1.7 & 5.1 \\
& $b$ & 1 & 3 & $\beta$ & 0 & 2.1 & $b$ & $\beta$ & 0 & 5.1 \\
& & & & $\gamma$ & 0 & 2.1 & $a$ & $\gamma$ & 0 & 5.1 \\
\hline 2 & $a$ & 0.9 & 2.6 & $\alpha$ & 0.7 & 1.7 & $b$ & $\alpha$ & 1.7 & 4.3 \\
& $b$ & 1 & 2.6 & $\beta$ & 0.5 & 1.5 & $b$ & $\beta$ & 1.5 & 4.1 \\
& $c$ & - & - & $\gamma$ & 0 & 1.5 & $a$ & $\gamma$ & 0 & 4.1 \\
& $e$ & 0 & 2.4 & & & & $e$ & $\beta$ & 0 & 3.9 \\
\hline 3 & $a$ & 1.5 & 2.1 & $\alpha$ & 0.7 & 1.3 & $b$ & $\alpha$ & 1.7 & 3.3 \\
& $b$ & 1 & 2 & $\beta$ & 0.5 & 0.9 & $b$ & $\beta$ & 1.5 & 2.9 \\
& $e$ & 0 & 1.5 & $\gamma$ & 0.2 & 0.6 & $a$ & $\gamma$ & 1.7 & 2.7 \\
& & & & & & & $e$ & $\beta$ & 0 & 2.4 \\
\hline 4 & $a$ & 1.5 & 1.7 & $\alpha$ & 0.8 & 0.9 & $b$ & $\alpha$ & 2 & 2.3 \\
& $b$ & 1.2 & 1.4 & $\beta$ & 0.5 & 0.7 & $b$ & $\beta$ & 1.7 & 2.1 \\
& $e$ & 0 & 0.6 & $\gamma$ & 0.2 & 0.4 & $a$ & $\gamma$ & 1.7 & 2.1 \\
& & & & & & & $e$ & $\beta$ & 0 & 1.5 \\
\hline
\end{tabular}

Table 2: Interleaved Execution example

Note that entity $c$ was pruned (line 13), as it does not participate in any package. After 2 more steps, in step 4 , package $e, \beta$ is ascertained to not be the top package, and it is pruned. This results in the pruning of entity $e$ as well. In the final step (not shown), package scores are fully disambiguated, and package $b, \alpha$ is determined to be the top package with score 2.1 .

\subsection{Extensions}

Informed Access Scheduling: The Interleaved algorithm described above is very efficient, without requiring any estimations. However, it is still not optimal. Consider a problem instance where, in order to identify the top 5 packages, 500 SA's are required on one inverted list, and only 10 on all the others. Since Interleaved performs SA's in a round robin fashion, 500 SA's will be performed on all lists before the algorithm terminates. It could thus help performance if accesses were scheduled taking properties of the underlying data distribution into account, so that fewer accesses are performed till termination.

Techniques for scheduling accesses in an informed manner have been proposed in [7], in the context of standard top-k query processing. It is only natural to investigate the extent to which such methods may be beneficial to our setting as well. Utilizing such techniques, one could try to effectively allocate SA's to inverted lists, so as to reduce the total number of required accesses. Using statistics on i) the score distribution of every inverted list and ii) the distribution of documents on every inverted list matching every entity, one may try to adapt the KBA framework from [7] to the entity package finder setting. Periodically, the adapted KBA framework can be used to estimate the "Benefit" that some number of SA's on a given list will have on processing, and select the most "beneficial" allocation of SA's to inverted lists ("Benefit" here is a quantity highly correlated with the remaining processing effort, i.e. highly "beneficial" SA's lead to shorter expected processing times).

Optimizing the schedule of SA's requires posing a number of queries over the materialized statistics. Due to our setting involving aggregation, compared to the setting in [7], informed scheduling requires that orders of magnitude more such queries be posed. Moreover, it requires that additional independence assumptions be made, leading to lower accuracy; hence it is expected to be less useful in reducing processing overhead. These expectations are consistent with our empirical observations, on our adaptation of KBA. On average, more than half of query time was spent on informed schedul- 
ing, as opposed to actual query processing; moreover, the amount of query processing effort was not significantly reduced. For these two reasons, we do not further pursue an informed scheduling approach in this work. Further details on our adaptation of KBA can be found in [3].

Further extensions: More generally, we note that Interleaved is amenable to further extensions of the entity package finder problem, such as taking into account individual user preferences; we discuss such extensions in [3].

\section{EVALUATION}

\subsection{Analysis}

As introduced, the Interleaved algorithm has two potential sources of performance benefits: interleaved pruning of entities and tight bounding of package scores. In this section, we propose two models that capture the intuition behind these sources, and help in comparing Interleaved with Layered. To effectuate this comparison, we use a hypothetical, idealized instantiation of the Layered algorithm, by providing it with an oracle for the exact values of all $D_{i}$ for the query being processed. To highlight this fact, we refer to this oracle instantiation of Layered as Layered ${ }^{D_{i}}$

\subsubsection{Benefits of interleaved pruning}

To capture the benefits of interleaved pruning, we introduce the notion of an entity-package ranking coefficient of a dataset. Most top-k query processing approaches (recast in entity package finder terms) assume that top packages tend to be composed of top entities. The entity-package ranking coefficient embodies, in a sense, the quantitative relationship between these; i.e. how many top entities are needed for every top package. As we will shortly see, this relationship strongly affects the effectiveness of interleaved pruning.

Whereas top packages tend to be composed of top entities, the converse is clearly not true; an entity $e$ with a mediocre score can be part of a top package, and an entity $e^{\prime}$ of the same type, with a higher score than $e$, might not be part of any. Let $E_{i}$ denote the set of all entities of the latter kind wrt. a query (i.e. top entities of type $T_{i}$, which nevertheless do not participate in any top package). Layered $^{D_{i}}$ expends unnecessary effort to fully process entities in $E_{i}$, by computing the top $D_{i}$ entities of each type independently of the resulting packages. Interleaved pruning, however, provides Interleaved the ability to avoid this overhead. We therefore expect that, the more entities in all $E_{i}$ that exist wrt. a query, the greater the performance improvements due to interleaved pruning. Observe that the number of such entities is strongly correlated with the total number of top entities, $D_{i}$, that need to be examined, as only a fraction of all entities examined will participate in some top package. Finally, note that all $D_{i}$ are positively correlated with the number of top packages required, since the more top packages that need to be identified, the more entities will have to be examined. Thus, we can quantify the effects of interleaved pruning by examining the expected number of entities that need to be examined per answer package identified, i.e. the expected value of $\frac{D_{i}}{k}$.

We quantify this value as follows: For a given corpus, and for every entity package finder query $W$, let $k$ be the number of top packages requested by $W$. As per definition, the rank of the lowest scoring entity of type $T_{i}$ that needs to be computed, in order to identify the top $k$ packages, is $D_{i}$. We define the entity-package ranking coefficient for this case, $c_{i}\left(T_{i}, W, k\right)$, as $c_{i}\left(T_{i}, W, k\right)=\frac{D_{i}}{k}$. Essentially, $c_{i}$ provides a measure of the number of entities of type $T_{i}$ that need to be calculated, in order to identify an additional top package; in other words, a measure of the necessary amount of pro- cessing per result package. In general $c_{i}$ will depend on many factors, such as the corpus, the query being processed, and the number of top packages requested.

Let $P(W, k)$ be the probability that query $W$ is issued, requesting a number $k$ of top packages (this probability may be derived from a known querylog, or via a uniformity assumption). We define the entity-package ranking coefficient of our entire dataset $C$, as a random variable, distributed according to the following distribution:

$$
\operatorname{Prob}(C=x)=\frac{1}{n} \sum_{\forall T_{i}, W, k} P(W, k) \cdot\left[\left|c_{i}\left(T_{i}, W, k\right)=x\right|\right]
$$

where $[|A|]=1$ if $\mathrm{A}$ is true, and 0 otherwise

Given the discussion presented above wrt. interleaved pruning, we expect that in every dataset, the relative performance of Interleaved versus Layered ${ }^{D_{i}}$ to be positively correlated with the expected value of $C$. That is, for larger expected values of $C$, we expect the performance gains of Interleaved versus the idealized Layered $^{D_{i}}$ to increase, due to the effects of interleaved pruning. We validate this trend in sec. 5.2, using micro-experiments which measure local expected values of $C$ in a dataset.

\subsubsection{Benefits of tight bounding}

We subsequently introduce the notion of entity association probability, that illuminates the performance benefits due to tight bounding. Entity association probability embodies a notion of known association "density", i.e. what fraction of possible associations in fact hold. We shortly discuss how this measure captures the performance benefits of tight bounding.

Recall that Interleaved utilizes known associations to provide tight bounds on the maximum score a package not yet encountered can have, MaxUnseenPkg. Tight bounding results from ensuring that the best package not yet encountered only consists of entities not yet encountered. Thus its score will be an aggregate of the scores, MaxUnseen $T_{i}$, of these entities (eqn. 2), as opposed to an aggregate of the scores of the best entities of each type and one of MaxUnseen $T_{i}$ (eqn. 1). The tightness of these bounds, and hence the effectiveness of the tight bounding scheme, depend on the probability, $P_{a s s o c}$, that $n$ arbitrary entities of different types are associated (i.e. form a valid package). As this likelihood increases, the value of information obtainable by known associations decreases, and tight bounding approaches the bounding scheme used by Layered ${ }^{D_{i}}$. To illustrate this point, observe that knowing which packages are valid is not very useful for bounding if a large fraction of possible packages are valid. Note that, by definition, $P_{\text {assoc }}=\{\#$ of known associations $\} / \Pi_{i}\left\{\#\right.$ of entities of type $\left.T_{i}\right\}$

On the other hand, Layered ${ }^{D_{i}}$ bounds MaxUnseenPkg in a conservative manner, as it cannot exploit known associations. Moreover, the number of top entities it calculates per type, $D_{i}$, is not dependent on the resulting packages. Thus, by increasing $P_{a s s o c}$, the only component affected in Layered ${ }^{D_{i}}$ is the rank-aware join operation. The latter is expected to terminate earlier for larger $P_{a s s o c}$, as $P_{a s s o c}$ corresponds to the join selectivity.

Thus, we expect the relative performance of Interleaved versus Layered $^{D_{i}}$ to be negatively correlated with $P_{a s s o c}$; i.e. for smaller values of $P_{a s s o c}$, we expect the performance gains of Interleaved versus the idealized Layered ${ }^{D_{i}}$ to increase, in part due to tight bounding. We validate this trend in sec. 5.2.

\subsection{Experimental Evaluation}

The Interleaved algorithm processes entity package finder queries without relying on knowledge or estimates of $D_{i}$. Layered requires 


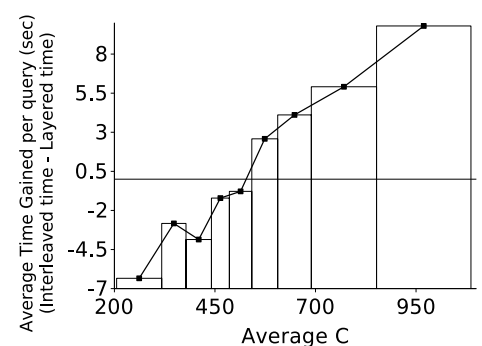

(a) Comparison to Layered $^{D_{i}}$ over $C$

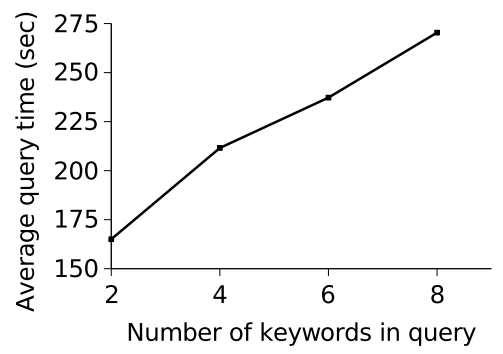

(d) Scalability vs \# keywords

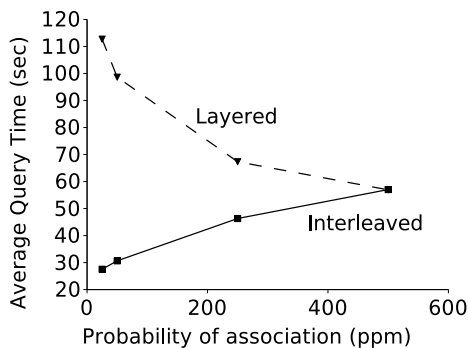

(b) Comparison to Layered ${ }^{D_{i}}$ over $P_{a}$

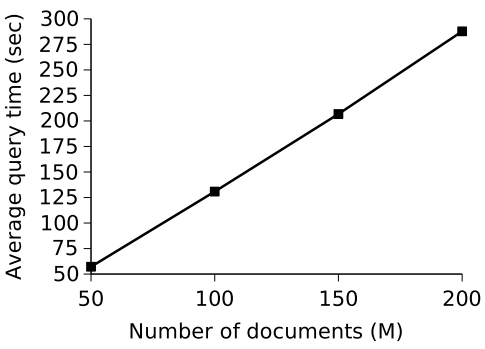

(e) Scalability vs \# documents

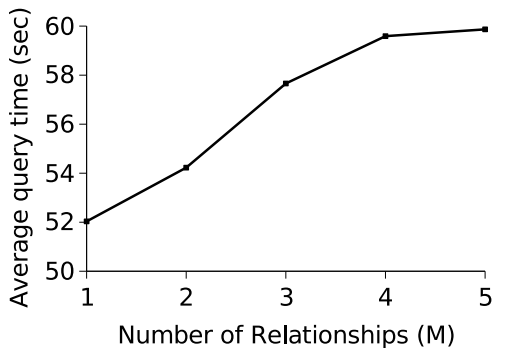

(c) Scalability vs \# document-entity relationships

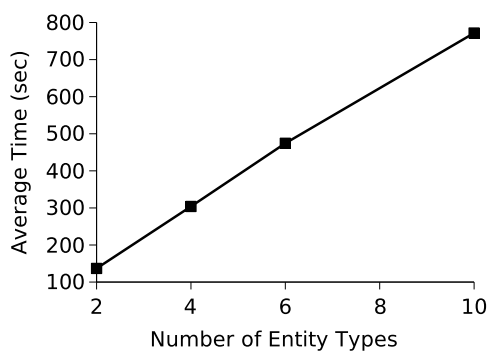

(f) Scalability vs \# entity types

Figure 3: Experimental Evaluation

these values, and their estimation, as shown in sec. 3.2, incurs an unreasonably high runtime and/or storage overhead. To effectuate a comparison between the two algorithms, and demonstrate the performance benefits of our proposed techniques, we compared Interleaved with Layered ${ }^{D_{i}}$, an idealized instantiation of Layered (i.e. Layered with an oracle for the exact values of all $D_{i}$ ). In order to implement the latter, the exact values of $D_{i}$ for every query were precalculated in a brute force manner.

Our experiments, on large-scale synthetic datasets show that Interleaved is about as efficient, and in practical cases even more efficient, than this idealized instantiation of Layered, thus manifesting the effectiveness of our techniques. Moreover, they validate the trends predicted in sec. 5.1. Further experiments demonstrate the efficiency and scalability of our approach, on both real and synthetic datasets.

In order to stress our algorithm on large-scale datasets, we generated large-scale synthetic corpora, denoted $S Y N T H$. Unless otherwise noted, all $S Y N T H$ corpora contained 50M documents, two types of entities, $10 \mathrm{~K}$ entities of each type and $5 \mathrm{M}$ document-entity relationships per entity type. Every keyword appeared in a varying fraction of all documents, ranging in 0.5-0.05. The queryload used, unless otherwise noted, consisted of 50 queries with 1-3 keywords per entity type, requesting the top 1-10 answers. For generating these corpora, all data distributions (document scores, documententity relationships, entity associations, query sizes, etc.) were uniform. Our experiments used static entity associations, however we note that using dynamic associations, as described in sec.2.1 does not affect our results; this is due to entity associations being accessed via the same API, regardless of whether they are static or dynamic.

To demonstrate the applicability and efficiency of our approach, we also experimented on a large corpus of real data, $R E A L$. We utilized data from BlogScope ([6]), an analysis and visualization tool for the blogosphere, currently monitoring over $28 \mathrm{M}$ blogs and over 400M blog posts. We used all indexed posts made in the 10day period between June 11th and 21st (excluding spam, and posts in languages other than English), resulting in over 3.7M documents. From these documents, we extracted 600K Named Entities of 9 different types, using an Information Extraction tool developed inhouse at the University of Toronto. In this way we extracted over 4.1M document-entity relationships. Entity associations were determined to hold between pairs of entities with statistically significant co-occurences in these blog posts, for a total of $110 \mathrm{~K}$ associations. Whereas $R E A L$ represents only a 10 day sample of discussions in the blogosphere, it serves to demonstrate that our approach can be efficiently applied on real-world, large-scale corpora.

We implemented both Layered ${ }^{D_{i}}$ and Interleaved in Java 1.6. As scoring functions (sec. 2.3) we used $F_{a g g}=\sum, F_{c o m b}=$ $\min , F_{p k g}=\sum$; this choice of scoring functions favors packages with entities that are, on average, most relevant to all query keywords. We note that other choices of scoring functions, embodying different semantics, are possible (subject to the loose constraints detailed in sec. 2.3); experiments with different scoring functions yielded similar performance trends. Our implementation maintained document-entity relationships and known associations in main memory; as discussed in sec 2.1 this is a reasonable assumption, even for very large corpora. All our experiments were executed on a machine with an Intel Core 2 Duo CPU, operating at $2.93 \mathrm{GHz}$, and 4GB of memory; our experiments utilized only one $\mathrm{CPU}$ core. In all experiments we report query running time, measured from the moment a query is issued until answers are reported to the user. We do not take into account the small runtime overhead of system initialization, as it occurs only once, regardless of the number of queries processed.

For validation purposes, we also compared our algorithms with an approach that used only RDBMS technology. Specifically, we stored the $S Y N T H$ corpus as indexed tables in a relational database (MySQL 5.5), and wrote our entity-package finder queries in SQL. We expect this approach to be highly inefficient, as it needs to calculate precise scores of every entity and package, followed by a selection of the top-k packages. Indeed, when executing our test queries, this approach had average query execution time up to an 
order of magnitude larger than our proposed Interleaved algorithm, depending on query parameters. For this reason, we do not further consider such RDBMS-based approaches, but focus instead on approaches with early termination and pruning properties (Interleaved and Layered ${ }^{D_{i}}$ ).

\subsubsection{Comparison with Layered ${ }^{D_{i}}$}

We first present an evaluation of the relative performance of Interleaved and an instantiation of Layered utilizing an oracle to obtain precise $D_{i}$ values, termed Layered ${ }^{D_{i}}$. We stress that this algorithm (Layered ${ }^{D_{i}}$ ) is provided as a point of comparison, and is not practically realizable (in practice, obtaining precise values of $D_{i}$ is not possible, and estimating them incurs unreasonable storage and/or runtime overheads, see sec.3.2). In our first comparative experiment, we varied the number of known associations in the $S Y N T H$ corpus, from $2.5 \mathrm{~K}$ to $500 \mathrm{~K}$, corresponding to $P_{a s s o c}$ of $25 \cdot 10^{-6}$ to $500 \cdot 10^{-6}$, and executed a queryload of 50 queries, each with 1-5 keywords per entity type, using both Interleaved, and Layered $^{D_{i}}$ (Recall that $P_{\text {assoc }}$ denotes the probability that $n$ arbitrary entities of different types are associated. Due to its semantics, we expect it to have very low values in practice. To provide a sense of perspective with respect to the values of $P_{a s s o c}$ tested, we note that observed values for $P_{a s s o c}$ in $R E A L$, the real-world corpus we used, ranged from $4.2 \cdot 10^{-6}$ to $21 \cdot 10^{-6}$. Thus, this experiment stresses Interleaved well beyond the operational parameters we typically expect to encounter.). In fig. 3(b) we show average query time for both Layered ${ }^{D_{i}}$ and Interleaved. As one can see, Interleaved outperforms Layered ${ }^{D_{i}}$ for all practical values of $P_{a s s o c}$, with performance gains of up to $76 \%$. Moreover, one can observe the trends predicted in sec. 5.1.2, namely that the performance of Interleaved increases, and that of Layered ${ }^{D_{i}}$ decreases, for lower values of $P_{a s s o c}$, due to the effects of tight bounding. Finally, we observe a roughly equal performance (Interleaved being $0.18 \%$ slower than Layered ${ }^{D_{i}}$ ), for $P_{\text {assoc }}=500 \cdot 10^{-6}$. Even though this is an unreasonably high value (in view of the observed $P_{\text {assoc }} \in\left[4.2 \cdot 10^{-6}, 21 \cdot 10^{-6}\right]$ in $R E A L$, as discussed above), we subsequently focus on it, to evaluate the performance benefits of interleaved pruning.

In our second comparative experiment, we evaluate the performance benefits of interleaved pruning. We expect Interleaved to relatively outperform Layered ${ }^{D_{i}}$ due to interleaved pruning, in cases with higher entity-package ranking coefficient, $C$, i.e. when top packages also require entities that are not among the top (see sec. 5.1.1). To validate this expectation, we utilized the $S Y N T H$ corpus, and executed a queryload of 500 queries, each with 1-5 keywords per entity type, using both Interleaved, and Layered ${ }^{D_{i}}$. As previously noted, the parameter $P_{a s s o c}$ was chosen to ensure a roughly equal performance, on average, of Interleaved and Layered ${ }^{D_{i}}$. Each query execution is a micro-experiment, where local expected values of $C$ in the dataset can be measured (as $a v g_{i}\left(\frac{D_{i}}{k}\right)$, where averages are computed over all entity types $T_{i}$, for the given query). We measured the performance benefits of interleaved pruning as Time Gained, which we define as \{execution time using Interleaved \{execution time using Layered $\left.{ }^{D_{i}}\right\}$. We grouped our observations using an equi-depth histogram on the local measured values of $C$, and report average Time Gained per query in fig. 3(a). We observe the trend predicted in sec. 5.1.1, thus validating our previous analysis. As a note, recall that we set $P_{a s s o c}$ to a "break-even" point between Interleaved and Layered ${ }^{D_{i}}$; had we set it to a lower value, such as observed in real data, fig. 3(a) would be completely favorable towards Interleaved.

\subsubsection{Scalability}

Having shown significant performance benefits of our proposed algorithm, Interleaved, over the oracle baseline Layered ${ }^{D_{i}}$, we next evaluate its scalability and efficiency, using large-scale synthetic corpora. Note that these are significantly larger, wrt. all operating parameters, than corpora one would expect in practice; as we subsequently demonstrate, using a real dataset, performance in practice is orders of magnitude better (sub-second average query time - cf. sec. 5.2.3).

Number of document-entity relationships: The number of document - entity relationships in a corpus is an important factor affecting query processing performance, as it influences the early pruning/termination capabilities of Interleaved, wrt. document score aggregation. To test the scalability of our approach, in this experiment we varied the number of document-entity relationships in the $S Y N T H$ corpus, from $1 \mathrm{M}$ to $5 \mathrm{M}$ per entity type, and executed our typical query workload using Interleaved. We show average query execution time in fig. 3(c), and observe that Interleaved gracefully scales to a large number of document-entity relationships.

Number of keywords in query: In this experiment we used the $S Y N T H$ corpus, and executed four query workloads of 50 queries each, using Interleaved. We varied the number of keywords in each query from 1 to 4 keywords per entity type (i.e. between 2 to 8 keywords per query). Average query execution time, shown in fig. $3(d)$, demonstrates that Interleaved scales gracefully with respect to the number of keywords in a query. We note that, typical user keyword queries involve a small number of keywords, a trend that we expect carries across to entity-package finder queries; in practice, we expect a typical workload to involve fewer keywords per query than in this experiment.

Number of documents: The number of documents contained in a corpus naturally affects performance, but is less crucial to performance than other parameters. Observe that, all other things being equal, scaling the number of documents in a corpus will increase I/O overhead, and the number of probes to document-entity relationship tables, but will not significantly affect other query processing components (e.g. rank-aware aggregation or join). We verify this trend by varying the number of documents in the $S Y N T H$ corpus, from $50 \mathrm{M}$ to $200 \mathrm{M}$ per entity type, and executing our typical query workload using Interleaved. Fig. 3(e) shows average query processing time, demonstrating a graceful, near-linear scaleup trend with respect to the number of documents in the corpus. This trend validates our expectations that Interleaved can efficiently scale up to very large document collections.

Number of entity types: In this experiment we varied the number of entity types in the SYNTH corpus, from 2 to 10 , and executed our typical query workload using Interleaved. We observed a near-linear scaleup in average query execution time (shown in fig. $3(f)$ ), demonstrating the scalability of our approach wrt. the number of entity types involved in a query. Note that, intuitively, an actual user query is expected to involve only a small number of types, and certainly fewer than 10; as in previous experiments, we chose to stress our algorithm with operating parameters significantly larger than in practice, to observe its trends wrt. scalability.

\subsubsection{Experiments with real data}

To demonstrate the applicability of our techniques on real data, we also utilize the $R E A L$ corpus described above. For every different kind of pairwise entity associations (e.g. Person A is associated with Company C, e.g.2 Band B is associated with Person D), we executed a query workload containing 200 queries, each with 1-5 keywords per entity type, using both Layered ${ }^{D_{i}}$ and Interleaved. Query keywords were randomly chosen from a list of adjectives most commonly used in English. 
The relative performance of Layered ${ }^{D_{i}}$ and Interleaved on real data validates our expectations from synthetic data (fig. 3(b)). Specifically, given that the $R E A L$ corpus exhibits values of $P_{a s s o c}$ significantly lower than those shown in fig. 3(b), we expect Interleaved to outperform Layered ${ }^{D_{i}}$ by a large margin. Indeed, when executed on the $R E A L$ corpus, Interleaved was more than one order of magnitude faster than Layered ${ }^{D_{i}}$.Moreover, Interleaved processed each query in under $1.5 \mathrm{sec}$; average execution time ranged from $0.25 \mathrm{sec}$ to $0.5 \mathrm{sec}$ per query, depending on the scenario of pairwise associations being tested. Overall, average query processing time using Interleaved was under $0.34 \mathrm{sec}$. We observe that our proposed algorithm is able to efficiently answer entity package finder queries on large, real-world corpora, validating our observations obtained from experimentation on synthetic corpora.

\section{RELATED WORK}

The entity package finder problem, presented in this work, belongs to the general area of top-k query processing. However, standard top-k techniques (e.g. [10]) do not apply, due to the document score aggregation that needs to take place. [9] and [5] proposed algorithms for calculating top-k over aggregation; however, these do not consider joins, and hence cannot be used for solving the entity package finder problem. Moreover, the techniques presented therein cannot be efficiently adapted to our problem, as the resulting algorithm would rely on estimations with very high runtime and/or storage overhead. Such estimation problems, albeit in much simpler settings, are discussed in [16]. The techniques proposed in this work cannot be efficiently applied in the entity package finder setting; the reason is that document score aggregation introduces an added complexity for providing the requisite statistics for estimation. Another related work is [2], which discusses methods for efficiently estimating properties of joins. However, these methods only apply to primary key-foreign key joins, and cannot thus be applied to our estimation problem, which involves a more general kind of joins.

Another related line of works deals with rank-aware join algorithms (e.g. [11], [15]), that efficiently compute top-k over joins. Our approach extends the scope of these frameworks, to include rank-aware aggregation. General rank-aware query processing systems have been extensively studied in the literature (e.g. [14], and its extensions [12], [18]). These works, however, do not discuss aggregation, and cannot thus be applied in the entity-package finder setting. A rank-aware query processing system capable of top-k query processing over joins and aggregation is proposed in [13], but the techniques it presents assume that joins occur before aggregation (e.g. as is typically the case in SQL queries). These semantics are not compatible with the entity package finder problem (where joins need to be performed on top of aggregated results), and the techniques proposed in this work cannot be efficiently applied to our setting.

Scheduling accesses in an informed, data-adaptive manner, for increased performance, has been investigated in [7], in the context of the Threshold Algorithm ([10]). An adaptation of such techniques to our setting is not practical, as it has a large runtime overhead, due to properties of our setting.

\section{CONCLUSIONS}

In this work, we introduced the class of entity package finder queries. We examined algorithms resulting from adaptations of previous work, and we proposed Interleaved, an efficient algorithm to process such queries, by devising early pruning and termination strategies, in the presence of joins and aggregations, that do not depend on any estimates. We demonstrate the efficiency and scalability of our approach analytically and by experiments, on both real and synthetic large-scale data.

\section{REFERENCES}

[1] Opencalais. http://www.opencalais.com. Retrieved on June 23, 2008.

[2] S. Acharya, P. B. Gibbons, V. Poosala, and S. Ramaswamy. Join synopses for approximate query answering. In SIGMOD Conference, pages 275-286, 1999.

[3] A. Angel, S. Chaudhuri, G. Das, and N. Koudas. Ranking objects based on relationships and fixed associations. Tech.report, 2008. Available at http://www.cs.toronto.edu/ albert/docs/acdk-edbt09.pdf.

[4] D. E. Appelt and D. Israel. Introduction to information extraction. In IJCAI Tutorial, 1999.

[5] N. Bansal, S. Guha, and N. Koudas. Ad-hoc aggregations of ranked lists in the presence of hierarchies. In SIGMOD Conference, 2008.

[6] N. Bansal and N. Koudas. Blogscope: A system for online analysis of high volume text streams. In $V L D B$, pages 1410-1413, 2007.

[7] H. Bast, D. Majumdar, R. Schenkel, M. Theobald, and G. Weikum. Io-top-k: Index-access optimized top-k query processing. In $V L D B$, pages 475-486, 2006.

[8] K. D. Bollacker, C. Evans, P. Paritosh, T. Sturge, and J. Taylor. Freebase: a collaboratively created graph database for structuring human knowledge. In SIGMOD Conference, pages 1247-1250, 2008.

[9] K. Chakrabarti, V. Ganti, J. Han, and D. Xin. Ranking objects based on relationships. In SIGMOD Conference, pages 371-382, 2006.

[10] R. Fagin, A. Lotem, and M. Naor. Optimal aggregation algorithms for middleware. In PODS, 2001.

[11] I. F. Ilyas, W. G. Aref, and A. K. Elmagarmid. Supporting top-k join queries in relational databases. In $V L D B$, pages 754-765, 2003.

[12] I. F. Ilyas, W. G. Aref, A. K. Elmagarmid, H. G. Elmongui, R. Shah, and J. S. Vitter. Adaptive rank-aware query optimization in relational databases. ACM Trans. Database Syst., 31(4):1257-1304, 2006.

[13] C. Li, K. C.-C. Chang, and I. F. Ilyas. Supporting ad-hoc ranking aggregates. In SIGMOD '06: Proceedings of the 2006 ACM SIGMOD international conference on Management of data, pages 61-72, New York, NY, USA, 2006. ACM.

[14] C. Li, K. C.-C. Chang, I. F. Ilyas, and S. Song. Ranksql: Query algebra and optimization for relational top-k queries. In SIGMOD Conference, pages 131-142, 2005.

[15] K. Schnaitter and N. Polyzotis. Evaluating rank joins with optimal cost. In PODS, pages 43-52, 2008.

[16] K. Schnaitter, J. Spiegel, and N. Polyzotis. Depth estimation for ranking query optimization. In $V L D B$, pages 902-913, 2007.

[17] A. Singhal. Modern information retrieval: A brief overview. IEEE Data Eng. Bull., 24(4):35-43, 2001.

[18] M. A. Soliman, I. F. Ilyas, and K. C.-C. Chang. Top-k query processing in uncertain databases. In ICDE, pages 896-905, 2007. 